\title{
Are You Working Vigorously? Adaptation and Validation of the Thai Version of Shirom-Melamed Vigor Scale
}

\author{
Watcharaporn Boonyasiriwat, Paradee Srisuwannatat, and Vipavee Puttaravuttiporn \\ Faculty of Psychology, Chulalongkorn University, Bangkok, Thailand
}

\begin{abstract}
upporting the application of positive psychology in the workplace, the Shirom Melamed Vigor Scale $S$ (SMVS), which assesses vigorous feelings at work, was adapted to the Thai context. The Thai SMVS contains 14 items as in the original scale and was validated using a sample of 585 employed participants in Thailand. Results of a confirmatory factor analysis are consistent with the conceptual model of vigour as consisting of physical strength, cognitive liveliness, and emotional energy, with a suggestion that a 13-item scale can also be used. As expected, vigour has been found to be conceptually distinct from some similar constructs, and independent from job burnout. Vigour also showed unique predictive effects on employees' health. These results support the previously-proposed conceptual framework of vigour and indicate crucial roles of positive emotion in the work setting. In concordance with a positive organisational psychology approach, applications of the Thai vigor scale are discussed with respect to its potential for enhancing employees' wellbeing and productivity.
\end{abstract}

Keywords: vigour, Shirom-Melamed Vigor Scale, positive emotion, employee, scale adaptation

Positive emotions have long been identified as an important factor for individuals to obtain favourable outcomes (e.g., resources and skills) and flourish in life (e.g., Fredrickson, 1998, 2001). Vigour, as a kind of positive emotion at work, has recently been proposed to play an important role in organisational settings (Shirom, 2011). To be vigorous means that individuals possess feelings of having physical strength, cognitive liveliness, and affective energy (Shirom, 2004, 2010, 2011), which has been hypothesised to predict positive physical and mental wellbeing of employees as well as major work outcomes (Shirom, 2011). Typically measured using the Shirom-Melamed Vigor Scale (SMVS; Shirom, 2004), accumulating evidence suggests that vigour might actually be worth investigating both in and out of the workplace setting (Shirom, Toker, Berliner, Shapira, \& Melamed, 2008; Shirom, Toker, Jacobson, \& Balicer, 2010; Shirom, Toker, Melamed, Berliner, \& Shapira, 2013; Steele et al., 2012). For instance, Shirom and colleagues (Shirom et al., 2008; Shirom, Toker, Jacobson et al., 2010; Shirom et al., 2013) found that feeling vigorous at work predicted better self-rated health (Shirom et al., 2008), as well as lower risk for diabetes (Shirom, Toker, Jacobson et al., 2010) and hyperlipidemia among healthy employees (Shirom et al., 2013). For work-related outcomes, vigour was the strongest predictor of job performance above job involvement, perceived organisational support, and work engagement (Steele et al., 2012; see also Carmeli, Ben-Hador, Waldman, \& Rupp, 2009). After reviewing these researches, it seems logical that exploring the role of this positive affective state in Thai employees is important. Specifically, Thailand is known as the 'Land of Smiles', which implies that Thai people use their positive emotions in many life contexts. Indeed, Sanuk, or having fun, has been suggested to be central to Thai's way of working (Kislenko, 2004). Surprisingly, the role of positive emotion in the Thai workplace has been neglected, though research examining positive states such as hope and resilience have provided promising results in predicting crucial work outcomes such as job satisfaction, work happiness, and organisational commitment (Maturapodpong, 2012). Adaptation of a validated scale measuring vigour might help to explain the role of positive affect at work in Thai culture. Such research could increase our understanding of positive psychology applied to work in a collectivistic context where work-related goals and values are different from the individualistic one (Hofstede, 1980).

Address for correspondence: Watcharaporn Boonyasiriwat, Ph.D. Faculty of Psychology, Chulalongkorn University, 7th Fl. Boromratchonnani Sri Sattapat Bldg., Rama 1 Rd. Patumwan, Bangkok, Thailand.Email: watcharaporn.p@chula.ac.th 
Therefore, the primary objective of the current study was to present the adaptation of the SMVS in the Thai language in a Thai population.

\section{Conceptualisation of Vigour at Work}

Vigour, as defined in the work context, consists of feelings that one is physically (i.e., has physical energy to handle tasks at work), emotionally (i.e., able to show sympathy and empathy to coworkers and customers), and cognitively strong (i.e., have flowing thought processes and mental agility at work; Shirom, 2004). Conceptualising vigour as an affective construct sets it apart from other similar constructs. According to Schaufeli, Salanova, González-Romá, and Bakker (2002), vigour is not actually a separate construct but falls within the conceptualisation of 'work engagement'. Vigour as part of work engagement refers to a state of having high levels of energy, mental resilience, eagerness to put effort into work, and persistence in the face of work difficulty. In contrast, Shirom (2011) moved away from work engagement per se and conceptualised vigour as based purely on positive affect, which can also have motivational implications. He viewed vigour to engage in work as being confounded by its possible 'consequences', which are resilience, willingness to work hard, and persistence. Shirom also argued that vigour and a contrasting state of burnout are conceptually independent from each other. In sum, he introduced vigour as a positive feeling or emotion that has its own place in the working context. Recently, Shirom (2011) proposed a theoretical model of vigour. The model predicts that resources available to employees (e.g., positive job characteristics, high-quality relationships with coworkers) will promote energetic feelings, resulting in a number of significant positive outcomes such as greater motivation at work, heightened job performance and organisational effectiveness, increased job satisfaction, and better physical and mental health.

\section{Measuring Vigour: The SMVS}

The 14-item SMVS developed by Shirom (2004) consists of three subscales reflecting three theoretical dimensions of vigour, namely: physical strength (PS; five items); cognitive liveliness (CL; five items); and emotional energy (EE, four items). Respondents rate how they feel each kind of strength within the past 30 working days on a 7point Likert scale ranging from 1 (never or almost never) to 7 (always or almost always). A global vigour score can be calculated by averaging item scores across each of the three subscales. The SMVS has good internal consistency with a Cronbach's alpha ranging between .87 to .92 for the global scale (Shirom, 2004; Shirom, Toker, Melamed et al., 2010; Shirom et al., 2013). The PS subscale has especially high internal consistency (Cronbach's alpha $=.93-.95$ ), as does the EE subscale (Cronbach's alpha $=.89-95$; Armon \& Shirom, 2011; Shirom, 2004; Shirom et al., 2008). Shirom (2004) reported a reliability of .72 for the CL sub- scale. A 13-item version with four items of CL has also been used, with reliabilities of .90-.92 (Armon \& Shirom, 2011; Shraga \& Shirom, 2009). The average intercorrelations between the three subscales have been reported with a medium relationship of $r=.44$ (Shirom, 2004).

Evidence supporting the validity of the SMVS is accumulating. Using a confirmatory factor analysis (CFA), Shirom and colleagues (Shirom, 2004; Shirom et al., 2008) found that the 14-item SMVS was best represented by the three proposed dimensions. In comparing the SMVS, work engagement (Utrecht Work Engagement [UWES]; Schaufeli et al., 2002), and engagement measure of Britt (1999) against each other, Wefald, Mills, Smith, and Downey (2012) reported that the SMVS fared better than the other two measures in predicting turnover intentions. The PS and CL subscales of the SMVS accounted for unique variance of turnover intentions after statistically controlling for job satisfaction and affective commitment, while none of the UWES subscales or Britt's measure did. Wefald and colleagues (2012) concluded that the SMVS is probably the best measure for operationalising the construct of work engagement. The SMVS also better predicts subsequent self-rated health (Shirom et al., 2008) and lower fibrinogen, which is an inflammatory biomarker (Shirom, Toker, Melamed et al., 2010), demonstrating good criterion validity. We therefore chose to adapt the 14-item SMVS vigour measure for the Thai language (referred to as T-SMVS).

In adapting the SMVS, we followed the guidelines by the World Health Organization (WHO, n.d.), which suggests three steps before producing a final version of an adapted scale: forward translation, expert panel backtranslation, and pretesting. We present the findings of the T-SMVS scale adaptation and validation in two steps: Step 1 describes the procedure we used for forward and backward translation, and item revision; Step 2 entails validation of the T-SMVS, including tests of the scale's construct, convergent, discriminant, and incremental validity. Although pretesting is recommended, we integrated this process into Step 1 by using experts to carefully evaluate the translated items and provide suggestions for the translation revision in order to best convey the meaning and form of the original items.

\section{Step I: Adaptation of the T-SMVS}

To construct the T-SMVS, we followed the steps outlined as follows:

Step 1: Investigation of concept. We reviewed literature on vigour and how it has been conceptualised as affect and as part of work engagement.

Step 2: Original statement translation. Thai native speakers then translated the SMVS from English to Thai. Comments and suggestions on the Thai version were obtained from 10 graduate students in psychology who were Thai native speakers and had reviewed literature on vigour. The translated scale was revised. 
Step 3: Back-translation. The T-SMVS was translated back into English by two Thai psychologists who had no prior knowledge of the concept of vigour or the SMVS. The first author then synthesised the two versions into a back-translation version.

Step 4: Expert committee rating. Two additional psychologists who are native English speakers independently assessed whether the back-translated English version was comparable to the original English version. One psychologist was an expert in cognitive and language learning, while the other was an expert in research methodology. Following suggestions of Sperber (2004), the back-translated English version was evaluated in terms of comparability of language and similarity of meaning. Comparability of language was assessed as whether words, phrases, and sentences were comparable between versions in terms of language form on a scale of 1 (not at all comparable) to 7 (extremely comparable). Similarity of the meaning ratings assessed whether meanings were similar between original and back-translated versions (equivalence of meaning even if the wording is not the same) as the corresponding item in the original scale $(1=$ not at all similar, $7=$ extremely similar). Ratings of the back-translated English items are shown in Table 1.

Step 5: Revision of problematic items. Items that were rated lower than the midpoint (lower than 4) in either comparability of language or similarity of meaning dimensions were modified. We found that items 1 , 'I feel full of pep', and 2, 'I feel I have physical strength', did not match the original items in terms of meaning. Items 5 , 'A feeling of vitality', and 10, 'A feeling of flow', suffered on both form and meaning. The Thai version of these items were modified.

Step 6: Repetition of back-translation. Only items 1, 2, 5 , and 10 were repeated in the back-translation process. Specifically, a psychologist who was a Thai native speaker with no background in the concept of vigour was asked to translate the items into English. The back-translated items were then subjected to the independent expert committee rating (same raters as in Step 4). Only item 10 was still evaluated as low on both form and meaning similarity (the back-translated item was 'thought fluidity'). The item was revised again in Thai, and Steps 5 and 6 were repeated for this item only until it passed the rating criteria on its third revision.

\section{Step II: Validation of the T-SMVS}

Dimensions of vigour. To validate vigour as a psychological workplace construct and to investigate how it relates to various emotional and health indicators, a number of additional measures were administered, including reports of emotional exhaustion, job burnout, vigour as a dimension of work engagement, positive and negative affect at work, work self-efficacy, self-esteem, psychological empowerment, self-reported physical health, as well as frequency of exercise. To assess the T-SMVS construct va- lidity, we assessed whether the scale was best represented by the three subscales of PS, CL, and EE according to the original construct outlined by Shirom (2004).

Hypothesis 1: A three-factor structure will be confirmed for the T-SMVS (PS, CL, and EE) to determine whether each item loads onto its corresponding dimension proposed by the original scale.

\section{Vigour as a Positive Affect}

According to Russell's (1980) circumplex model of affect predicting that each emotion is a product of valence and arousal dimensions combined in various degrees, vigour was postulated to locate on moderate activation (vs. deactivation) and pleasant (vs. unpleasant) quadrant (Shirom, 2004). Therefore, the T-SMVS overall vigour score should correlate moderately with positive feelings at work in general (not specific in terms of arousal level, but specific for work setting), indicating convergent validity. At the same time, vigour as an affect should also correlate moderately with general negative affects experienced at work (e.g., nervous, distressed), which could be evidence for the T-SMVS's discriminant validity.

Hypothesis 2a: The T-SMVS global vigour score will have a moderate positive correlation with other positive affects at work, and a moderate negative correlation with negative affects at work.

In arguing for distinctiveness of vigour as purely affective, Shirom $(2004,2011)$ proposed that vigour should not overlap with work engagement as the former is an affective state whereas the latter can be considered a motivational consequence of vigour. Moreover, vigour as a facet of work engagement encompasses not only a high level of energy, but also mental resilience and willingness to put effort into work, which might be consequences of being vigorous rather than the construct itself. Due to some overlap in conceptualising vigour in the two frameworks, previous research found that the two vigour constructs are strongly related, but still distinctive (Wefald et al., 2012).

Hypothesis 2b: The T-SMVS overall vigour will have a high positive correlation with vigour in work engagement.

\section{Vigour and Job Burnout}

The state of being vigorous at work might be viewed as the absence of being stressed or burnt out. In conceptualising vigour as an affect, Shirom $(2004,2011)$ pointed to research findings from stress and adaptation literature for adopting the bivariate approach that views vigour and burnout as two distinct constructs instead of two poles of a single emotional dimension. He argued that vigour belongs to a behavioural approach system that directs individuals toward expanding activity levels. Job burnout, expressed as emotional exhaustion, cynicism, and reduced professional efficacy (Maslach, Schaufeli, \& Leiter, 2001), in contrast, is viewed as a cause of behavioural avoidance 
Table 1

Average Ratings of Comparability of Language and Similarity of Interpretability of the Back-Translated T-SMVS as Compared to Original Items and Standardised Solutions by Confirmatory Factor Analysis for the Three-Factor Model

\begin{tabular}{|c|c|c|c|c|c|c|c|c|c|c|}
\hline \multirow[b]{3}{*}{ No. } & \multirow[b]{3}{*}{ Item } & \multicolumn{6}{|c|}{$\begin{array}{l}\text { Average ratings } \\
\text { (Range of score is } 1-7 \text { ) }\end{array}$} & & & \\
\hline & & \multicolumn{2}{|c|}{ Round 1} & \multicolumn{2}{|c|}{ Round 2} & \multicolumn{2}{|c|}{ Round 3} & \multicolumn{3}{|c|}{ Factor loadings } \\
\hline & & $\mathrm{COL}$ & $\mathrm{SI}$ & $\mathrm{COL}$ & $\mathrm{SI}$ & $\mathrm{COL}$ & $\mathrm{SI}$ & PS & $\mathrm{CL}$ & $\mathrm{EE}$ \\
\hline 1 & I feel full of pep & 4.00 & 2.50 & 7.00 & 6.50 & - & - & .76 & & \\
\hline 2 & I feel I have physical strength & 4.50 & 2.50 & 4.50 & 6.00 & - & - & .87 & & \\
\hline 3 & I feel vigorous & 4.50 & 5.50 & - & - & - & - & .90 & & \\
\hline 4 & I feel energetic & 7.00 & 7.00 & - & - & - & - & .90 & & \\
\hline 5 & A feeling of vitality & 3.50 & 3.00 & 6.50 & 6.50 & - & - & .84 & & \\
\hline 6 & I feel mentally alert & 5.00 & 4.50 & - & - & - & - & & .80 & \\
\hline 7 & I feel I can think rapidly & 5.50 & 4.50 & - & - & - & - & & .85 & \\
\hline 8 & I feel I am able to contribute new ideas & 6.00 & 6.50 & - & - & - & - & & .84 & \\
\hline 9 & I feel able to be creative & 6.50 & 6.50 & - & - & - & - & & .80 & \\
\hline 10 & A feeling of flow & 3.50 & 3.50 & 3.50 & 3.50 & 5.50 & 5.00 & & .84 & \\
\hline 11 & I feel able to show warmth to others & 6.00 & 6.50 & - & - & - & - & & & .72 \\
\hline 12 & I feel able to be sensitive to the needs of coworkers and customers & 6.50 & 6.50 & - & - & - & - & & & .75 \\
\hline 13 & I feel I am capable of investing emotionally in coworkers and customers & 6.00 & 4.50 & - & - & - & - & & & .86 \\
\hline 14 & I feel capable of being sympathetic to coworkers and customers & 5.50 & 4.50 & - & - & - & - & & & .83 \\
\hline
\end{tabular}

Note: Items shown are from the original English language Shirom-Melamed Vigor Measure (Shirom, 2004). COL = comparability of language, $\mathrm{SI}=$ similarity of interpretability, $\mathrm{PS}=$ physical strength, $\mathrm{CL}=$ cognitive liveliness, $\mathrm{EE}=$ emotional energy. Bold italic numbers are average ratings that are lower than midpoint of the rating scale (4, on 1-7 scale), indicating that the translated item needed revision.

system motivating individuals to withdraw from ongoing high job demands. Research examining the relationship between burnout and work engagement (with vigour as a subscale) provides evidence supporting the view that burnout and vigorous affect may be two different experiences rather than two ends of the same emotional dimension (Demerouti, Mostert, \& Bakker, 2010; GonzálezRomá, Schaufeli, Bakker, \& Lloret, 2006; Mäkikangas, Feldt, Kinnunen, \& Tolvanen, 2012).

Hypothesis 3: The T-SMVS vigour will not correlate with job burnout.

Central to the job burnout concept is emotional exhaustion (Maslach et al., 2001). If vigour is an affective state of being resourceful emotionally, physically, and cognitively, the opposite state would be emotional exhaustion, a chronic state of resource depletion or fatigue due to excessive job demands. According to the conservation of resources model (COR; Hobfoll, 1989), emotional exhaustion could be a result of an actual/perceived resource loss, which could lead to diminished job performance and subsequent voluntary turnover (Wright \& Cropanzano, 1998). Previous research (treating vigour and emotional exhaustion as part of work engagement and job burnout respectively), however, has documented a weakto-moderate negative relationship between vigour and emotional exhaustion (Demerouti et al., 2010; GonzálezRomá et al., 2006; Mäkikangas et al., 2012). The weak relationship may indicate that, like vigour and job burnout, vigour and emotional exhaustion might not be opposite ends of energy levels at work, but perhaps related experiences that can occur simultaneously within a person. Investigating the relationship between vigour and emo- tional exhaustion will provide evidence with regard to the bivariate view of vigour and job burnout. (Mäkikangas et al., 2014; Shirom et al., 2013).

Hypothesis 4: The T-SMVS vigour will have a weak to moderate negative correlation with emotional exhaustion.

\section{Vigour and Its Antecedents}

To prove its distinctiveness, vigour should display discriminant validity with some positive but different constructs such as self-esteem, self-efficacy, and being psychologically empowered. Unlike vigour, self-esteem represents a view of the self that varies in degrees of favourableness. Self-efficacy represents cognitive, not affective, assessment of one's abilities to accomplish a task. Self-efficacy has also been proposed to be a component of psychological empowerment, a motivational state of being competent, seeing meaning in work, and having autonomy at work (Spreitzer, 1995). According to Shirom's $(2004,2011)$ vigour framework, these three constructs should be viewed as resources that are internal to the individual and should lead employees to subsequently feel vigorous. It was thus expected that vigour would have positive relationships, albeit only small to moderate in size, with these constructs, as described in the following hypotheses:

Hypothesis 5a: The T-SMVS vigour will have a weak-tomoderate positive correlation with self-esteem.

Hypothesis 5b: The T-SMVS vigour will have a weak-tomoderate positive correlation with self-efficacy specific to work domain. 
Hypothesis 5c: The T-SMVS vigour will have a weakto-moderate positive correlation with psychological empowerment.

\section{Vigour and Health Consequences}

Finally, to validate the T-SMVS, we included a test of its relationship with physical health. According to Shirom's (2011) framework of antecedents and consequences of vigour, vigour is predictive of greater physical health. This prediction is based on research linking vigour to greater self-rated health (Shirom et al., 2008; Shirom, Toker, Jacobson et al., 2010).

Hypothesis 6: The T-SMVS vigour will correlate positively with self-rated health as indicated by self-report of physical health status and frequency of exercise, controlling for age, gender, and income.

\section{Method}

\section{Participants}

A total of 585 participants were recruited from five different work organisations in Bangkok, Thailand $(68.9 \%, n=$ 403 women, $31.1 \%, n=182 \mathrm{men}$ ), including a pharmaceutical supplier and petrochemical, telecommunications and information technology, statutory audit, and legal companies. Participants completed a number of scales in the Thai language in paper-and-pencil format. The mean age of the participants was 29.98 years $(S D=5.95)$. The mean length of service in current employment was 4.84 years $(S D=5.10)$. Characteristics of participants are shown in Table 2.

\section{Measures}

Vigor. The 14-item back-translated T-SMVS was used to measure vigorous feelings at work. Respondents were asked to report how frequently they experience each kind of vigour dimensions (PS, CL, and EE) in the past 30 working days on a 7-point frequency scale ranging from 1 (never or almost never) to 7 (always or almost always). Responses on the 14-items were summed to yield a global vigour score, with higher scores indicating higher levels of vigour.

Emotional exhaustion. A nine-item emotional exhaustion scale of Saralumba (2009) was used. The scale assesses the extent to which a person experiences physical and emotional fatigue due to job demands. Participants were asked to indicate their degree of agreement with statements such as 'I feel like I have overworked', using a 6 -point scale ranging from 1 (never) to 6 (always). This scale has been validated among a sample of Thai employees by Saralumba (2009) and shown to have high reliability $(\alpha=.90)$. Cronbach's alpha for the present sample was .88.

Job burnout. A 16-item measure of job burnout adapted from Schaufeli, Leiter, Maslach, and Jackson's (1996) job burnout scale by Kleebbua (2009) was used to gauge employees' experience of burnout at work using three subscales: exhaustion (5 items,
Table 2

Demographic Characteristics of All Participants $(N=585)$

\begin{tabular}{|c|c|c|c|c|c|}
\hline Variable & $n$ & $\%$ & $M$ & $S D$ & Range \\
\hline Age & & & 29.98 & 5.95 & $18-56$ \\
\hline Work duration (year) & & & 4.84 & 5.10 & $0.8-44$ \\
\hline \multicolumn{6}{|l|}{ Gender } \\
\hline Male & 182 & 31.11 & & & \\
\hline Female & 403 & 68.89 & & & \\
\hline \multicolumn{6}{|l|}{ Education } \\
\hline High school or less & 23 & 3.93 & & & \\
\hline Post-high school & 12 & 2.05 & & & \\
\hline Bachelor's & 407 & 69.57 & & & \\
\hline Postgraduate & 137 & 23.42 & & & \\
\hline Not reported & 6 & 1.03 & & & \\
\hline \multicolumn{6}{|l|}{ Organisational rank } \\
\hline Operation level & 475 & 81.20 & & & \\
\hline High-level manager & 80 & 13.68 & & & \\
\hline Middle-level manager & 24 & 4.10 & & & \\
\hline Low-level manager & 2 & 0.34 & & & \\
\hline Not reported & 4 & 0.68 & & & \\
\hline \multicolumn{6}{|c|}{ Salary (monthly, in Thai baht) } \\
\hline Less than 10,000 & 11 & 1.88 & & & \\
\hline $10,000-50,000$ & 461 & 78.80 & & & \\
\hline $50,001-100,000$ & 39 & 6.67 & & & \\
\hline 100,001 or more & 4 & 0.68 & & & \\
\hline Not reported & 70 & 11.97 & & & \\
\hline \multicolumn{6}{|l|}{$\begin{array}{l}\text { Fringe benefits (monthly, } \\
\text { in Thai baht) }\end{array}$} \\
\hline Less than 10,000 & 303 & 51.79 & & & \\
\hline $10,000-50,000$ & 196 & 33.51 & & & \\
\hline $50,001-100,000$ & 15 & 2.56 & & & \\
\hline 100,001 or more & 17 & 2.91 & & & \\
\hline Not reported & 54 & 9.23 & & & \\
\hline
\end{tabular}

$\alpha=.83$ ), cynicism (5 items, $\alpha=.90$ ), and professional efficacy (6 items, $\alpha=.84$ ). Participants indicated their agreement with statements such as 'I have grown to be less interested in my job since I started it' on a 7-point scale ranging from 0 (never) to 6 (everyday). Cronbach's alpha in our sample was high: exhaustion (five items, $\alpha=.88$ ), cynicism (five items, $\alpha=.87$ ), and professional efficacy (six items, $\alpha=.94$ ).

Vigour facet of work engagement. A six-item vigour in work engagement was assessed using Jirawit-Olan's (2011) scale adapted from the UWES (Schaufeli et al., 2002). Participants responded to statements such as 'When I wake up in the morning, I feel I want to go to work'. Their responses were assessed on a 5-point scale ranging from 1 (strongly disagree) to 5 (strongly agree). This scale has been validated in a Thai employee sample by Jirawit-Olan (2011) with good reliability $(\alpha=.86)$. Cronbach's alpha for the present sample was .77.

Positive and negative affect at work. A 20-item scale from Thisaphak (2005), which was adapted from Watson, Clark, and Tellegen's (1988) positive and negative affect scale, was used to assess the extent to which participants usually experience both kinds of affect specifically at work. The scale consists of 10 positive affect items (e.g., inspired, active) and 10 negative affect items (e.g., fear, nervous). Each item was rated on a 7-point scale ranging from 1 (very 
slightly or not at all) to 7 (extremely). A composite score for positive (negative) affect was computed by adding ratings of the 10 items. The scale has been validated in a sample of Thai employees and showed high reliability ( $\alpha=.93$ for positive affect items and $\alpha=.92$ for negative affect items). Cronbach's alpha for the present sample was .95 and .92 for positive and negative affect respectively.

Work self-efficacy. An 18-item scale developed by Pattarawit (2010) was used to gauge participants' beliefs in their capability to overcome obstacles and accomplish their goals at work. Participants were asked to indicate their agreement with statements such as 'I feel like I can handle difficulties in my job' on a 5-point scale ranging from 1 (strongly disagree) to 5 (strongly agree). The scale's reliability has been established among a sample of Thai employees $(\alpha=.86)$. Cronbach's alpha for the current sample was .93.

Self-esteem. Global evaluation of self was measured using a 24 -item, self-esteem scale developed by Srisrual (2004) based on Rosenberg's Self-Esteem Scale (Rosenberg, 1965). Participants were asked to indicate their degree of agreement with statements such as 'I like being myself' on a 5-point scale ranging from 1 (strongly disagree) to 5 (strongly agree). This scale has been validated in a Thai sample by Srisrual (2004) and demonstrated high reliability $(\alpha=.91)$. Cronbach's alpha for the current sample was .84 .

Psychological empowerment. A 24-item measure of the state of being intrinsically motivated by a task was used to assess cognitive manifestations of heightened intrinsic job motivation (Boonyaprasop, 2011). Participants responded to statements such as 'The job I am doing is meaningful for me' on a 5-point scale ranging from 1 (strongly disagree) to 5 (strongly agree). This scale has been validated in a Thai sample and demonstrated high reliability $(\alpha=.97)$. Cronbach's alpha for the current sample was .95 .

Physical health. Participants reported their general health relative to people their age on a 5-point scale ranging from 1 (poor) to 5 (excellent). Responses from these two questions were summed to derive a self-report physical health score (correlation between the two items, $r=.82$, $p<.001)$.

Exercise. A single question asking participants how frequent they had exercised in the last two weeks $(1=$ none, $5=$ very often) was used to assess physical activeness.

Data collection was deployed via 11 IRB-approved research projects that, during their scale development step, involved measuring vigour among Thai employees. These projects were independent from each other while all of them shared a goal of jointly developing the T-SMVS. Data for the T-SMVS was therefore collected from a variety of organisations in the Bangkok area of Thailand. All participants completed the T-SMVS, while each subgroup also received different sets of scales: participants 1-
70 completed the positive and negative affect at work and the psychological empowerment scales; 71-140 received the emotional exhaustion, vigour in work engagement, and self-esteem scales; $141-120$ and 351-420 received the burnout and work self-efficacy scales; 211-280 reported their self-esteem, physical health, and exercise, and filled out the T2 T-SMVS 2 weeks later; 281-350 reported their physical health and exercise; 421-490 completed emotional exhaustion and vigour in the work engagement scales; 491-560 reported their physical health and exercise, the positive and negative affect at work and psychological empowerment; and 561-585 only responded to the T-SMVS and no other scale.

\section{Data Analysis}

To test hypothesis 1 about the factor structure of the T-SMVS, a confirmatory factor analysis (CFA) was performed to compare model fit between the hypothesised and an alternative models. To test hypotheses 2 to 5 regarding convergent and discriminant validity, correlations were used. Finally, hypothesis 6 was tested using hierarchical regression analyses.

\section{Criterion for Model Testing}

Prior to testing our model using factor analysis, the following model fit criterion was used to determine good fit between the data and the model: the root mean square error of approximation (RMSEA) $<0.06$ for good fit, the standardised root mean residuals (SRMR) $<0.05$, the comparative fit index (CFI) $>0.95$, the adjusted goodness-of-fit index (AGFI) $>.92$, and the incremental fit index (IFI) $=0.95$.

\section{Incremental Validity of the T-SMVS}

Hierarchical regression analyses were conducted to determine whether the T-SMVS vigour had unique predictive ability when other similar constructs were controlled. In all analyses, the controlled constructs included demographic variables of age, gender, and income level entered as a single block in the first step. The T-SMVS vigour score was entered in the second step. If the T-SMVS vigour was conceptually distinctive from other constructs, it should significantly explain some proportion of the variance of the outcome measures in addition to similar constructs (after the other constructs were statistically controlled).

\section{Results}

Means, SDs, and Cronbach's alphas of all measures are shown in Table 3. Participants generally reported a moderate level of vigour $(M=4.69, S D=0.92, N=585)$, moderately good health $(M=2.98, S D=0.90, n=210)$, and had exercised occasionally in the last 2 weeks $(M=$ $2.24, S D=1.11, n=210$ ). When separated by dimension, as shown in Table 4, participants reported somewhat high levels of EE $(M=5.27, S D=1.20)$ and moderate levels of PS and CL $(M=4.35, S D=1.27$, and $M=4.56, S D=$ 
Table 3

Means, Standard Deviations, and Correlations of All Variables With the T-SMVS

\begin{tabular}{lllllll}
\hline Variable & $n$ & Range & $M$ & $S D$ & Alpha & Correlation with T-SMVS \\
\hline 1. Vigour (T-SMVS) & 585 & $1-7$ & 4.69 & 0.92 & .93 & - \\
2. Emotional exhaustion & 140 & $1-6$ & 3.23 & 0.89 & .88 & $-.43^{* * *}$ \\
3. Job burnout 3 facets & 140 & $1-6$ & 4.04 & 0.77 & $.88-.94$ & -.11 \\
4. Vigour facet of work engagement & 140 & $1-5$ & 3.67 & 0.57 & .77 & $.64^{* * *}$ \\
5. Positive affect at work & 140 & $1-7$ & 4.76 & 1.10 & .93 & $.74^{* * *}$ \\
6. Negative affect at work & 140 & $1-7$ & 3.02 & 1.13 & .92 & $-.33^{* * *}$ \\
7. Work self-efficacy & 140 & $1-5$ & 3.75 & 0.66 & .93 & $.65^{* * *}$ \\
8. Self-esteem & 140 & $1-5$ & 3.68 & 0.40 & .84 & $.49^{* * *}$ \\
9. Psychological empowerment & 140 & $1-5$ & 3.56 & 0.60 & .95 & $.61^{* * *}$ \\
10. Physical health & 210 & $1-5$ & 2.98 & 0.90 & - & $.44^{* * *}$ \\
11. Exercise & 210 & $1-5$ & 2.24 & 1.11 & - & $.33^{* * *}$ \\
\hline
\end{tabular}

Note: ${ }^{* * *} p<.001$

Table 4

Descriptive Statistics for the Three T-SMVS factors $(N=585)$

\begin{tabular}{llllcllllll}
\hline & & & & & & & & \multicolumn{3}{c}{ Correlations } \\
\cline { 5 - 8 } \cline { 5 - 8 } & No. of items & $M$ & $S D$ & Skewness & Kurtosis & Alpha & 1 & 2 & 3 \\
\hline 1. Physical strength & 5 & 4.35 & 1.27 & .11 & .13 & .93 & - & & \\
2. Cognitive liveliness & 5 & 4.56 & 1.29 & -.15 & .20 & .91 & $.76^{* * *}$ & - & \\
3. Emotion energy & 4 & 5.27 & 1.20 & -.46 & .06 & .87 & $.55^{* * *}$ & $.63^{* * *}$ & - \\
\hline
\end{tabular}

Note: Range of scores is $1-7$. ${ }^{* * *} p<.001$.

1.29 respectively). The three subscales had intercorrelations between .55 to .76 , with an average intercorrelation of .64. The CL and EE subscales had negatively skewed distributions, whereas the PS subscale was positively skewed. The skewness and kurtosis of all subscales, however, were within range to assume a normal distribution.

\section{Hypothesis Testing for Scale Validation}

To test hypothesis 1 , the T-SMVS was subjected to a confirmatory factor analysis $(N=585)$ using LISREL version 8.72 (Jöreskog \& Sörbom, 2004) in order to test whether the three-factor model fit the data. Although there is no theoretically competing model for vigour's component, one might argue that vigour represents one type of positive affect. The hypothesised model was thus tested against an alternative model in which all 14 items were restricted to load onto a single latent vigour variable (a one-factor model). The correlated three-factor model fit the data reasonably well although the chi-square value was significant, indicating bad fit with the data, $\chi^{2}(74, N=585)=$ $342.26, p<.001$. However, because chi-square is sensitive to large sample size (Bentler \& Bonett, 1980), other fit indices were considered. Other commonly used fit indices indicated that the three-factor model fit the data reasonably well: RMSEA $=.08(<0.06$ for good fit $)$, the SRMR $=.04(<0.05)$, CFI $=.98(>0.95)$, AGFI $=.89$ $(>.92)$, and IFI $=0.98$ ( $>.95$ and is very close to one). All standardised loadings were higher than .70, indicating high correlations between each item and their corresponding factor. Factor loadings of each item are provided in Table 1. Each T-SMVS item loaded on its subscale in accordance with the original SMVS. The one- factor model, on the other hand, showed poor fit with the data, $\chi^{2}(77, N=585)=1816.92, p<.001$, RMSEA $=$ $.24, \mathrm{CFI}=.88$, SRMR $=.11$, AGFI $=.46$, IFI $=.88$. Fit statistics shown in Table 5 indicate better model fit for the correlated three-factor model on all fit indices with the value of $\chi^{2} / d f$ of the three-factor model closer to 3 . A chisquare difference test indicated that the three-factor model fit significantly better than did one-factor, $\Delta \chi^{2}(\Delta 3)=$ 1474.66, $p<.001$, supporting the three-factor structure hypothesis (H1). For the hypothesised model, maximum modification index indicated potential cross-loading for item 6 in CL dimension ('I feel mentally alert') such that it is recommended to load on the PS component, which is inconsistent with the measurement framework. Once this item was removed from the model, the fit was improved: $\chi^{2}(62, N=585)=261.96, p<.001$, RMSEA $=$ $.07, \mathrm{CFI}=.98, \mathrm{SRMR}=.06, \mathrm{AGFI}=.91, \mathrm{IFI}=.98 . \mathrm{In}$ summary, supporting hypothesis 1 , the CFA and model comparison results indicate that the three-factor model is better with acceptable fit to the data, and thus support the construct validity of the translated version of the SMVS. Removal of item 6 seems to slightly improve model fit.

\section{Reliabilities of the T-SMVS}

Internal consistency. As shown in Table 3 and Table 4, the T-SMVS exhibited excellent internal consistency for the overall scale $(\alpha=.93)$. Each subscale also showed high internal consistency $(\alpha=.93, .91$, and .87 for PS, CL, and EE, respectively).

Test-retest. A subsample of 140 participants completed the T-SMVS after a 2-week interval. The T-SMVS vigour 


\begin{tabular}{|c|c|c|c|c|c|c|c|c|}
\hline Model & $d f$ & $x^{2}$ & $\chi^{2} / d f$ & $\mathrm{CFI}$ & RMSEA & SRMR & AGFI & $\mathrm{IFI}$ \\
\hline One factor & 77 & 1816.92 & 23.60 & .88 & .24 & .11 & .46 & .88 \\
\hline Three factor & 74 & 342.26 & 4.62 & .98 & .08 & .04 & .89 &. \\
\hline Three factor without item 6 & 62 & 261.96 & 4.23 & .98 & .07 & .04 & .91 & .98 \\
\hline
\end{tabular}

Note: $\chi^{2}$ values are significant at $p<.001$. CFI = comparative fit index; RMSEA = root-mean-square error of approximation SRMR = standardized root mean residuals; $A G F I=$ adjusted goodness-of-fit index; IFI = incremental fit index.

scores from the two tests were highly correlated, $r=.77$, $p<.001$, indicating good test-retest reliability of the scale.

\section{Convergent and Discriminant Validity of the T-SMVS}

To demonstrate convergent validity of the 14 -item TSMVS, scores from the scale were hypothesised to correlate moderately with measures of positive affect at work and vigour dimension of work engagement. For each subset, correlations between T-SMVS and the other variables were investigated. Correlations are shown in Table 3. Vigour scores measured by the T-SMVS had significant positive correlations with positive affect experienced in the work setting ( $r=.71$ for PS, .59 for CL, .55 for EE, $p<.001$, and $r=.74, p<.001, n=140$ for the entire scale), and vigour in work engagement ( $r=.63$ for PS, .59 for CL, .29 for EE, $p<.001$, and $r=.64, p<.001, n=140$ for the entire scale). Thus, hypotheses $2 \mathrm{a}$ and $2 \mathrm{~b}$ were relatively well supported, indicating convergent validity of the T-SMVS.

Results also show that the T-SMVS total score had moderate correlations with the negative constructs of emotional exhaustion $(r=-.43, p<.001, n=140)$ and negative affect at work $(r=-.33, p<.001, n=140)$, as well as self-esteem $(r=.49, p<.001, n=140)$. The T-SMVS total score correlated more strongly with work self-efficacy $(r=.63, p<.001, n=140)$ and psychological empowerment $(r=.61, p<.001, n=140)$. The T-SMVS did not correlate with job burnout scale $(r=-.11, p=.18$, $n=140$ ). These results lend support to hypotheses $2 \mathrm{a}-5 \mathrm{c}$ and thus indicates discriminant validity of the T-SMVS.

\section{Incremental Validity}

Results of the hierarchical regression are shown in Table 6.

Exercise. Using a subset of data $(n=70,19$ men and 51 women, $\left.M_{\text {age }}=34.00, S D=7.95\right)$, the T-SMVS was tested to investigate whether it could predict amount of exercise after controlling for positive affect at work, psychological empowerment, and demographic variables. Results indicated that the T-SMVS significantly predicted amount of exercise ( $\beta=.39, p=.01)$ after controlling for age $(\beta=.30$, $p=.02)$, gender $(\beta=-.09, p=.45)$, income $(\beta=-.15, p=$ $.19)$, positive affect at work $(\beta=.36, p=.02)$, and psychological empowerment $(\beta=-.11, p=.45)$. Addition of the T-SMVS vigour score significantly increased the variance accounted for by the model from $30 \%$ to $37 \%\left(\Delta R^{2}=.07\right.$, $\Delta F(1,61)=7.29, p=.01)$.
Physical health. A subset of data $(n=210,59$ men and 151 women, $M_{\text {age }}=29.70, S D=6.13$ ) were analysed to test whether T-SMVS vigour scores could predict health ratings after controlling for demographic variables. Results showed that the T-SMVS score $(\beta=.41, p<.001)$ significantly predicted physical health after controlling for age $(\beta=.06, p=.46)$, gender $(\beta=-.19, p=.02)$, and income $(\beta=-.09, p=.27)$. Adding the T-SMVS vigour score significantly increased the variance accounted for by the model from $4 \%$ to $20 \%, \Delta R^{2}=.16, \Delta F(1,161)=31.68$, $p<.001$, in support of hypothesis 6 .

\section{Discussion}

The construct of vigour as an affect, a combination of being physically, mentally, and emotionally energetic at work, has been proposed to explain various positive organisational consequences (Shirom, 2011). The present study is the first to adapt and validate Shirom's (2004) measure of vigour for Thai employees. Findings support all of the hypotheses, indicating that the developed T-SMVS was a reliable tool and that vigour is a robust affective construct in workplace setting.

A CFA confirmed that the T-SMVS has three dimensions that cover physical (PS), emotional (EE), and cognitive vigour (CL) as with its original scale. Each item also loaded appropriately on its proposed dimension. Although item 6 ('I feel mentally alert') and 10 ('A feeling of flow') have sometimes been excluded from the original scale, data from Thai employees shows that both items have good loadings on the corresponding CL factor. Interestingly, like the original English SMVS, the Thai version suggests that modification of item 6 could improve the model. Exclusion or modification of item 6 is thus potentially beneficial for further developing the scale, though keeping the item is not considered problematic. An issue to be considered before removing item 6 would be whether the removal could affect coverage of the CL subscale representing states of having flowing thought processes and mental agility at work. Further CFA with larger samples would also be beneficial for testing the item's contribution to the scale.

The findings also indicate that the T-SMVS is internally consistent and psychometrically robust. We obtained strong internal consistency of the five-item CL dimension $(\alpha=.91)$. The EE subscale had the lowest alpha of .87, which is still considered to be high. Indeed, the present data collected from various types of organisations 


\begin{tabular}{|c|c|c|c|c|c|c|c|c|c|c|}
\hline \multirow[b]{3}{*}{ Step and predictor variable } & \multicolumn{10}{|c|}{ Criterion variable } \\
\hline & \multicolumn{5}{|c|}{ Physical health $(n=210)$} & \multicolumn{5}{|c|}{ Exercise $(n=70)$} \\
\hline & $B$ & $S E B$ & $\beta$ & $R^{2}$ & $\Delta R^{2}$ & $B$ & $S E B$ & $\beta$ & $R^{2}$ & $\Delta R^{2}$ \\
\hline Step 1: & & & & .04 & & & & & $.30^{* * *}$ & \\
\hline Age & .01 & .01 & .06 & & & .04 & .02 & $.30^{*}$ & & \\
\hline Gender & -.38 & .16 & $-.19^{*}$ & & & -.22 & .28 & -.09 & & \\
\hline Income & -.06 & .05 & -.09 & & & -.10 & .08 & -.15 & & \\
\hline Positive affect at work & - & - & - & - & - & .36 & .14 & $.36^{*}$ & & \\
\hline Psychological empowerment & - & - & - & - & - & -.21 & .28 & -.11 & & \\
\hline Step 2: & & & & $.20 * * *$ & $.16^{* * *}$ & & & & $.37^{* *}$ & $.07^{*}$ \\
\hline T-SMVS vigor & .41 & .07 & $.41^{* * *}$ & & & .46 & .17 & $.39 * *$ & & \\
\hline
\end{tabular}

Note: ${ }^{*} p<.05,{ }^{* *} p<.01,{ }^{* * *} p<.001$.

shows higher overall internal consistency $(\alpha=.93)$ than those previously reported (Shirom, 2004; Shirom, Toker, Melamed et al., 2010; Shirom et al., 2013). Other analyses also indicate appropriate convergent and discriminant validity of the T-SMVS. Scores from the T-SMVS were moderately related to other kinds of positive affect at work, as well as vigour as a component of work engagement. At the same time, the T-SMVS vigour is distinguishable from self-esteem, self-efficacy, emotional exhaustion, and psychological empowerment. Interestingly, we obtained insignificant findings between T-SMVS vigour and job burnout. This provides evidence in favour of the bivariate view of vigour and job burnout adopted by Shirom (2011). Specifically, findings from Thai employees provides evidence that positive affect of vigour is not similar to absence of job burnout. The two constructs might instead be viewed as two distinct states rather than two opposite ends of a single emotion. The present study, therefore, adds to the literature on the conceptualisation of vigor and job burnout.

Consistent with Shirom et al. (2008), the T-SMVS provided evidence for incremental validity by predicting important physical health outcomes from self-reported physical health and exercise, above and beyond the effects of other positive affect at work, psychological empowerment, and major demographic variables. These findings also provide evidence in support of Shirom's (2011) framework highlighting vigorous feelings as a distinctive affect at work that is a proximal predictor of positive work and health outcomes.

Some limitations of the present study, however, deserve attention. First, the test of incremental validity is limited to health outcomes, which were health status and frequency of exercise. Although previous studies have consistently documented the predictive effects of vigour on several physiological indicators of good health (e.g., Shirom, Toker, Jacobson et al., 2010; Shirom, Toker, Melamed et al., 2010; Shirom et al., 2013), findings with regard to predicting mental wellbeing and organisational outcomes are scarce (with the exception of Carmeli et al., 2009). Future investigations might provide more evidence for incremental validity of the T-SMVS on outcomes such as employees' subjective wellbeing and job performance. Second, related to the first issue, the current study presents tests of the T-SMVS' independent predictive ability on amount of exercise and self-report physical health, after demographic variables and a few correlated constructs were statistically controlled. However, we did not test effects of T-SMVS vigour against other similar constructs such as vigour in work engagement. Future investigations might add stronger evidence on uniqueness of the vigour construct. Third, convenient sampling was used in the survey, which resulted in a disproportionate number of females (approximately 2:1) in the overall sample. Although sample sizes were adequate for the majority of the tests, one for testing incremental validity on amount of exercise could be considered small $(n=70)$. Fourth, it should be noted that the T-SMVS was validated against a Thai version of Western scales, and such validations have not been published. However, the findings show expected relationships between those measures and the T-SMVS. Finally, all of our measures were self-report, potentially creating common method variance that might influence the results. Future research may consider utilising different sources of measurement besides self-report; for example, objective health rating by physician instead of selfrated health or employer's rating of behaviour or job outcomes in providing more evidence for the predictive effects of the T-SMVS. In addition, although the current study has employed some prevention of common method variance, that is, ensuring respondent anonymity that would help reduce evaluation apprehension during the survey completion, other remedies are also recommended (e.g., Podsakoff, MacKenzie, Lee, \& Podsakoff, 2003).

Despite the limitations of the present study, this successful adaptation of a vigour scale for a Thai context has some important applications. First, the introduction of the T-SMVS permits workplace vigour to be examined in the context of Thai culture. It may be the case that employee vigour matters more in collectivist (such as in Thailand) than individualist contexts for work productivity or other important outcomes for several reasons. As collectivist cultures place greater emphasis on group goals as compared to individualist cultures (Triandis, 1989), it is 
possible that vigour of employees in collectivistic cultures is used for achieving the goals of an organisation rather than those of an individual. Moreover, in collectivist cultures, emotional contagion occurs more frequently as individuals are influenced more strongly by the feelings of group members than are those in individualistic cultures (Ilies, Wagner, \& Morgeson, 2007). Thai people are also known as being fun loving at work. Such acceptance of displaying emotions at work should make emotional contagion occur more easily. Investigating how feelings of vigour or lack thereof might spread among employee groups could be a helpful tool to model, understand, and perhaps even improve employee morale, health, happiness, and productivity. In addition, describing how cultural systems function and interact to create vigour at work will be a major advancement for organisational and positive psychology.

Second, from an organisational psychology perspective, the T-SMVS permits investigation among Thai employees if vigorous feelings are central to various kinds of positive work-related outcomes as proposed by Shirom (2011). Findings would not only provide evidence to the framework, but will also inform management of how to achieve organisational goals based on positive psychology of supplying resources and creating positive feelings among employees. For instance, supervisors may enhance subordinates' vigorous feelings and reap the benefit of increased organisational citizenship behaviours if they support employees who have security and protection needs appropriately (Little, Nelson, Wallace, \& Johnson, 2011). Job performance may be enhanced via increased employees' vigour as they have a relational leadership (Carmeli et al., 2009). Expanding or replicating such studies in Thai employees would yield interesting findings from different cultural background that would promote a flourishing of research in positive emotions and positive organisational psychology, while also being useful for management.

Finally, with this Thai version of vigour, application of the measure within the workplace can be encouraged. Knowledge about employees' vigour can be utilised in various ways, for instance, as an indicator of employees' resource levels. Based on the COR theory (Hobfoll, 1989), vigorous feelings are expected to derive from various kinds of resources, both individual and work related (Shirom, 2011). Measuring vigour would reflect employees' readiness to utilise such resources to attain goals at work. If vigour is low, organisations may need to find out what the antecedents might be (e.g., negative leadership behaviour, low social support, ineffective reward practice), which could lead to improvement in management system and work environment. Feeling vigorous may be more imperative for some kinds of work more than the others, such as work or tasks that are related to creative problem solving or helping others (e.g., in customer service department), and actions that are associated with having positive moods (Isen \& Levin, 1972; Isen, Daubman, \& Nowicki, 1987). Knowing how much and in what context employ- ees experience vigour is an important piece of information for promoting such outcomes. The T-SMVS thus would be a useful tool for discovering more about antecedents and consequences of feeling vigorous at work in Eastern contexts.

\section{Acknowledgments}

This study was partially funded by the Center for Psychological Assessment, Faculty of Psychology, Chulalongkorn University. We gratefully thank all the scale's raters for the back-translation procedure. We also thank graduate students in the Applied Psychology, and the Industrial and Organizational Psychology Program at the Faculty of Psychology - Chantip Songkampol, Pirada Tipparat, Kornika Thanasetsakul, Chattamakarn Ruenaim, Sriruan Sripattanakul Hanbang, Anak Sopittanon, Wisakpa Phurikrai, Massalin Nimitsopon, Maneerat Vuthitannakul, Sukanlaya Yangsongjai - who enthusiastically assisted on data collection of the present study. We also thank Professor Emeritus Nonglak Wiratchai, $\mathrm{PhD}$, who provided advice on the statistical analyses of the study. In addition, we very much thank Damith Woods, $\mathrm{PhD}$, and Jason Ludington, $\mathrm{PhD}$, for a critical reading of and thoughtful comments on earlier versions of the manuscript. Finally, we are grateful for our reviewers who provided thoughtful comments and suggestions on improving this manuscript.

\section{References}

Armon, G., \& Shirom, A. (2011). The across-time associations of the five-factor model of personality with vigor and its facets using the bifactor model. Journal of Personality Assessment, 93, 618-627.

Bentler, P.M., \& Bonett, D.G. (1980). Significance tests and goodness of fit in the analysis of covariance structures. Psychological Bulletin, 88, 588-606.

Boonyaprasop, J. (2011). Relationships among psychological empowerment, perceived organizational support, and organizational commitment (Unpublished master's thesis). Chulalongkorn University, Bangkok, Thailand.

Britt, T.W. (1999). Engaging the self in the field: Testing the triangle model of responsibility. Personality and Social Psychology Bulletin, 25, 696-706.

Carmeli, A., Ben-Hador, B., Waldman, D.A., \& Rupp, D.E.(2009). How leaders cultivate social capital and nurture employee vigor: Implications for job performance. Journal of Applied Psychology, 94, 1553-1561.

Demerouti, E., Mostert, K., \& Bakker, A.B. (2010). Burnout and work engagement: A thorough investigation of the independency of both constructs. Journal of Occupational Health Psychology, 15, 209-222.

Fredrickson, B.L. (1998). What good are positive emotions? Review of General Psychology, 2, 300-319.

Fredrickson, B.L. (2001). The role of positive emotions in positive psychology: The broaden-and-build theory of positive emotions. American Psychologist, 56, 218-226. 
González-Romá, V., Schaufeli, W.B., Bakker, A.B., \& Lloret, S. (2006). Burnout and work engagement: Independent factors or opposite poles? Journal of Vocational Behavior, 62, 165174.

Hobfoll, S.E. (1989). Conservation of resources: A new attempt at conceptualizing stress. American Psychologist, 44, 513-524.

Hofstede, G. (1980). Culture's consequences: International differences in work-related values. Beverly Hills, CA: Sage.

Ilies, R., Wagner, D.T., \& Morgeson, F.P. (2007). Explaining affective linkages in teams: Individual differences in susceptibility to contagion and individualism-collectivism. Journal of Applied Psychology, 92, 1140-1148.

Isen, A.M., \& Levin, P.F. (1972). Effect of feeling good on helping: Cookies and kindness. Journal of Personality and Social Psychology, 21, 384-388.

Isen, A.M., Daubman, K.A., \& Nowicki, G.P. (1987). Positive affect facilitates creative problem solving. Journal of Personality and Social Psychology, 52, 1122-1131.

Jöreskog, K.G., \& Sörbom, D. (2004). LISREL 8.7 for Windows [Computer software]. Skokie, IL: Scientific Software International.

Jirawit-Olan, I. (2011). Effects of work self-efficacy and organizational culture on work engagement and subjective well-being: The mediating of flow at work (Unpublished master's thesis). Chulalongkorn University, Bangkok, Thailand.

Kislenko, A. (2004). Culture and customs of Thailand. Westport, CT: Greenwood Press.

Kleebbua, C. (2009). Development of the causal model of burnout at work: An application of the job demand-resource model. (Unpublished master's thesis). Chulalongkorn University, Bangkok, Thailand.

Little, L.M., Nelson, D.L., Wallace, J.C., \& Johnson, P.D. (2011). Integrating attachment style, vigor at work, and extra-role performance. Journal of Organizational Behavior, 32, 464484.

Mäkikangas, A., Feldt, T., Kinnunen, U., \& Tolvanen, A. (2012). Do low burnout and high work engagement always go hand in hand? Investigation of the energy and identification dimensions in longitudinal data. Anxiety, Stress \& Coping, 25, 93-116.

Mäkikangas, A., Kinnunen, S., Rantanen, J., Mauno, S., Tolvanen, A., \& Bakker, A. (2014). Association between vigor and exhaustion during the workweek: A person-centered approach to daily assessments. Anxiety, Stress and Coping, 27, 555-575.

Maslach, C., Schaufeli, W., \& Leiter, M. (2001). Job burnout. Annual Review of Psychology, 52, 397-422.

Maturapodpong, S. (2012). Hope, optimism, and resilience as predictors of performance, job satisfaction, work happiness, and organizational commitment (Unpublished master's thesis, Chulalongkorn University, Bangkok, Thailand).

Pattarawit, S. (2010). Relationships among career self-efficacy, goal commitment, and perception of career growth (Unpublished master's thesis). Chulalongkorn University, Bangkok, Thailand.

Podsakoff, P.M., MacKenzie, S.B., Lee, J.-Y., \& Podsakoff, N.P. (2003). Common method biases in behavioral research: A critical review of the literature and recommended remedies. Journal of Applied Psychology, 88, 879-90387.

Rosenberg, M. (1965). Society and the adolescent self-image. Princeton, NJ: Princeton University Press.

Russell, J.A. (1980). A circumplex model of affect. Journal of Personality and Social Psychology, 39, 1161-1178.

Saralumba, B. (2009). Relationship between emotional exhaustion and job performance: The multi-mediating effect of motivation (Unpublished master's thesis). Chulalongkorn University, Bangkok, Thailand.

Schaufeli, W.B., Leiter, M.P., Maslach, C., \& Jackson, S.E. (1996). Maslach Burnout Inventory - General Survey. In C. Maslach, S.E. Jackson, \& M.P. Leiter (Eds.), The Maslach Burnout Inventory manual (3rd ed.). Palo Alto, CA: Consulting Psychologists Press.

Schaufeli, W.B., Salanova, M., Gonzalez-Roma, V., \& Bakker, A.B. (2002). The measurement of engagement and burnout: A two sample confirmatory factor analytic approach. Journal of Happiness Studies, 3, 71-92.

Shirom, A. (2004). Feeling vigorous at work? The construct of vigor and the study of positive affect in organizations. In D.C. Ganster \& P.L. Perrewe (Eds.), Research in organizational stress and well-being.(vol. 3, pp. 135-164). Greenwich, CN: JAI Press.

Shirom, A. (2010). Feeling energetic at work: On vigor's antecedents. In A.B. Bakker \& M.P. Leiter (Eds.), Work engagement: A handbook of essential theory and research (pp. 69-84). New York, NY: Psychology Press.

Shirom, A. (2011). Vigor as positive affect at work: Conceptualizing vigor, its relations with related constructs, and its antecedents and consequences. Review of General Psychology, $15,50-64$.

Shirom, A., Toker, S., Berliner, S., Shapira, I., \& Melamed, S. (2008). The effects of physical fitness and feeling vigorous on self-rated health. Health Psychology, 27, 567-575.

Shirom, A., Toker, S., Jacobson, O., \& Balicer, R.D. (2010). Feeling vigorous and the risks of all-cause mortality, ischemic heart disease, and diabetes: A 20-year follow-up of healthy employees. Psychosomatic Medicine, 72, 727-733.

Shirom, A., Toker, S., Melamed, S., Berliner, S., \& Shapira, I. (2010). Vigor, anxiety, and depressive symptoms as predictors of changes in fibrinogen and C-reactive protein. Applied Psychology: Health and Well-Being, 2, 251-271.

Shirom, A., Toker, S. Melamed, S., Berliner S., \& Shapira, I. (2013). Burnout and vigor as predictors of the incidence of hyperlipidemia among healthy employee. Applied Psychology: Health and Well-being, 5, 79-98.

Shraga, O., \& Shirom, A. (2009). The construct validity of vigor and its antecedents: A qualitative study. Human Relations, 62, 271-291.

Sperber, A.D. (2004). Translation and validation of study instruments for cross-cultural research. Gastroenterology, 126, S124-S128.

Spreitzer, G.M. (1995). Psychological empowerment in the workplace: Dimensions, measurement, and validation. Academy of Management Journal, 38, 1442-1465. 
Srisrual, S. (2004). The influence of positive illusions on relationship satisfaction of dating couples (Unpublished master's thesis). Chulalongkorn University, Bangkok, Thailand.

Steele, J.P., Rupayana, D.D., Mills, M.J., Smith, M.R., Wefald, A., \& Downey, R.G. (2012). Relative importance and utility of positive worker states: A review and empirical examination. Journal of Psychology: Interdisciplinary and Applied, 146, 617-650.

Thisaphak, P. (2005). The effects of forgiveness and interpersonal commitment on psychological well-being. (Unpublished master's thesis). Chulalongkorn University, Bangkok, Thailand.

Triandis, H.C. (1989). The self and social behavior in differing cultural contexts. Psychological Review, 96, 506-520.
Watson, D., Clark, L.A., \& Tellegen, A. (1988). Development and validation of brief measures of positive and negative affect: The PANAS scales. Journal of Personality and Social Psychology, 54, 1063-1070.

Wefald, A.J., Mills, M.J., Smith, M.R., \& Downey, R.G. (2012). A comparison of three job engagement measures: Examining their factorial and criterion-related validity. Journal of Applied Psychology: Health and Well-being, 4, 67-90.

World Health Organization (WHO). (n.d.). Process of translation and adaptation of instruments. Retrieved from http://www.who.int/substance_abuse/research_tools/ translation/en/

Wright, T.A., \& Cropanzano, R. (1998). Emotional exhaustion as a predictor of job performance and voluntary turnover. Journal of Applied Psychology, 83, 486-493. 